Annuaire suisse de politique de développement

$7 \mid 1988$

Annuaire Suisse - Tiers Monde 1987/88

\title{
L'horlogerie suisse et les nouveaux pays industriels asiatiques
}

Die schweizerische Uhrenindustrie und die asiatischen Schwellenländer

Jean-François Blanc

\section{OpenEdition}

12 Journals

Édition électronique

URL : http://journals.openedition.org/aspd/1343

DOI : $10.4000 /$ aspd. 1343

ISSN : 1663-9669

Éditeur

Institut de hautes études internationales et du développement

Édition imprimée

Date de publication : 1 janvier 1988

Pagination : 203-221

ISSN : 1660-5934

\section{Référence électronique}

Jean-François Blanc, "L'horlogerie suisse et les nouveaux pays industriels asiatiques », Annuaire suisse de politique de développement [En ligne], 7 | 1988, mis en ligne le 05 avril 2013, consulté le 08 septembre 2020. URL : http://journals.openedition.org/aspd/1343; DOI : https://doi.org/10.4000/ aspd. 1343 


\title{
L'horlogerie suisse et les nouveaux pays industriels asiatiques
}

\author{
Jean-François Blanc
}

Zusammenfassung: Dle schwelzerlsche Uhrenlndustrle und dle aslatischen Schwellenländer

In diesem Beitrag untersucht der Verfasser die Zusammenhänge, die sich zwischen dem Umstrukturierungsprozess der Uhrenindustrie in der Schweiz und der Errichtung neuer Uhrenproduktions- und exportstätten in den Schwellenländem Asiens (Hongkong, Sudkorea, Taiwan und Singapur) ergeben, Er geht dabei von der Tatsache aus, dass der in den vergangenenen Jahren eingetretene technologische Wandel weltweit zu einer Umverteilung der Uhrenindustrie (Verlagerung in die Billiglohnländer) gefuhrt hat.

Im ersten Teil wird der Uhrenhandel zwischen der Schweiz und den asiatischen Schwellenlandern untersucht. Dabei werden folgende Tendenzen festgestellt. Ab 1970 bedeutende Einfuhren von Billiguhren und Uhrenbestandteilen aus den asiatischen Schwellenländem (und anderen asiatischen Lăndern). Bís 1980 umfan greiche schweizerischo Lieferungen an Uhrenbestandteilen in diese neuen Produktionsstătten, danach Rückgang dieses Trends. Seit einigen Jahren ein gewisser Wiederaufschwung des Schweizer Uhrenexports (vor allem elektronische Uhren), nach einem langwierigen Umstrukturierungsprozess der schweizerischen Uhrenindustrie.

Im zweiten Teil wird die Niederlassungspolitik der Schweizer Uhrenindustrie in den asiatischen Schwellenländern analysiert. An der Fallstudie Hongkong wird gezeigt, dass die Produktionsverlagerung zu einem relativ spatten Zeitpunkt und in beschränktem Ausmass erfolgte. Dies lässt sich durch die besondere Struktur der 
Schweizer Uhrenindustrie (langsames Vordringen der neuen Technologien, Rückgang der Marktpräsenz, starker Beschäftigungsrückgang in der Branche ) erklären.

In seinen Schlussfolgerungen führt der Verfasser am Beispiel Hongkongs eine Reihe von Gründen an, die eine nuancierte Bewertung der Bemühungen der Schweizer Uhrenindustrie zur Produktionsverlagerung in die asiatischen Schwellenländern ratsam erscheinen lassen. Er kommt zu dem Schluss, dass sich statt einer Opposition zwischen den jungen asiatischen Industrieländern und den traditionellen Industriestaaten derzeit eine Tendenz zur wachsenden internationalen Arbeitsteilung in der Uhrenindustrie abzeichnet; hierbei scheint Hongkong eine mittlere Position zwischen den modernen Forschungs- und Entwicklungszentren (Japan, Schweiz) und den "Wirtschaftszonen" der Billiglohnländer (China, Thailand, Malaysia und Philippinen) einzunehmen. 
Nul n'ignore que l'industrie horlogère mondiale aura été marquée, au cours de ces dix à quinze dernières années, autant par une profonde mutation technologique le passage de la micro-mécanique - que par un vaste mouvement de redistribution géographique de ses activités de production.

En effet, alors que la Suisse, à l'image d'ailleurs de la plupart des pays horlogers «traditionnelsw et à l'exception notable du Japon, connaissait un recul très sensible de son volume de production et d'exportations, un certain nombre de pays en Asie du Sud-Est, ceux-là mêmes que l'on appelle les "Nouveaux Pays Industriels» (PNI) asiatiques (1) - Hong Kong en tout premier lieu, mais aussi la Corée du Sud, Taiwan et Singapour -, se sont rapidement affirmés en tant que producteurs et exportateurs d'horlogerie.

Ceci est tellement vraique le part de ces pays à la valeur des exportations horlogères mondiales s'élevait à plus d'un tiers en 1984 -dont $24 \%$ pour la seule colonie de Hong Kong! - (2) et que leur production de montres et mouvements était estimée cette même année à plus de la moitié de la production mondiale exprimée en nombre de pièces - dont plus d'un tiers pour Hong Kong - (3). Al'inverse, la position de la Suisse, jadis largement dominante, n'a cessé de se réduire pour représenter, toujours en 1984, $25 \%$ environ des exportations horlogères en valeur-contre $54 \%$ en 1970 - et $6 \%$ de la production mondiale de montres et mouvements en volume - contre 38\% encore en 1974.

Ces quelques données pourraient alors laisser croire à un phénomène de transfert ou de "délocalisation " quasi-général et irréversible des activités productives horlogères en direction de pays à main-d'ceuvre abondante et bon marché. N'était-ce pas là l'effet d'une suite d'innovations qui, si elles ont donné lieu à une rapide substitution de nouveaux produits - les montres à quartz à affichage analogique ou digital - aux produits horlogers mécaniques, ont aussi considérablement simplifié, banalisé, la plupart des opérations d'assemblage, que ce soit des nouveaux composants horlogers (résonateurs à quartz, circuits intégrés, affichages à cristaux liquides...) ou des produits finis proprement dits (montres et mouvements). Cela ne signifiait-il pas également à plus long terme que les activités de production horlogère étaient amenées à disparaittre en pays industrialisés, en Suisse en particulier, si l'on excepte la fabrication de produits de haut de gamme ou de produits très sophistiqués?

A l'analyse, cependant, ce phénomène de “délocalisation» industrielle apparaît comme beaucoup plus modeste et surtout plus complexe qu'il n'y semblait à première vue, puisqu'il ne paraît avoir concerné en définitive que certaines opérations de production et certaines technologies et gammes de produits, et que, de plus, le développement de l'automation semble avoir fortement réduit au cours de ces dernières années l'avantage que représentait le bas niveau de salaires dans les Nouveaux Pays Industriels.

Sans pouvoir aborder ici, dans le cadre de cet article, l'ensemble des questions relatives à ce processsus de "délocalisation", nous nous limiterons à en souligner quelques aspects au sujet de l'horlogerie suisse en étudiant, premièrement, l'évolution des échanges horlogers entre la Suisse et les NPI asiatiques et, deuxièmement, la politique d'implantation industrielle des horlogers suisses 
en Asie du Sud-Est. Bien que très limitée, cette double approche nous permettra de conclure par quelques remarques sur la division internationale du travail qui paraît s'établir dans l'horlogerie.

\section{Les échanges horlogers entre la Suisse et les NPI asiatiques}

\section{Une industrie de moins en moins "autonome»}

Jusqu'à la veille des années 1970, l'industrie horlogère suisse demeure une industrie largement "autonome" dans le sens où elle produit par elle-même les diverses "consommations intermédiaires" (pièces détachées, boîtes de montres et autres pièces de l'habillement, ébauches ou mouvements non assemblés, mouvements terminés, etc.) dont elle a besoin, en même temps qu'elle paraît largement dominer le marché intérieur suisse en ce qui concerne la distribution de produits finis.

C'est du moins ce que tend à montrer la situation des importations horlogères suisses (voir tableau 1 en page suivante) dont le montant jusqu'en 1970 ne représente, par comparaison, qu'une très faible part de celui des exportations horlogères suisses. On constate en effet que ces importations se constituent alors pour l'essentiel de pièces détachées et de boîtes de montres que les industriels horlogers suisses se procurent, là aussi pour l'essentiel, auprès de pays voisins (République Fédérale d'Allemagne, France, Italie...). De fait, cette situation n'est pas nouvelle et correspond à la présence dans ces pays, en plus de fournisseurs indépendants, d'un certain nombre de sociétés filiales des entreprises horlogères suisses: ainsi en est-il à titre d'exemple du groupe ASUAG (Allgemeine Schweizerische Uhrenindustrie $A G$ ) qui, par le biais de ses différentes sociétés affiliées, contrôle des fabriques d'ébauches en France et en Allemagne Fédérale, des fabriques de pierres d'hologerie en Italie, etc.

Seule parmi les NPI asiatiques Hong Kong joue alors un rôle relativement significatif en tant que fournisseur de boîtes de montres principalement.

Quant aux importations de montres complètes, destinées à la distribution locale, elles ne portent que sur 130.000 pièces environ en 1970 , ce qui ne représente qu'une faible part de la consommation apparente de la Suisse.

De 1970 à 1985, on peut alors relever les évolutions suivantes:

- tout d'abord la forte croissance du montant total des importations qui représente désormais, en 1985 , plus de $12 \%$ de celui des exportations horlogères suisses. Cette pénétration du marché suisse par les produits étrangers reste certes de faible ampleur dans son ensemble, mais elle devient tout de même de plus en plus marquée en ce qui concerne certaines catégories de produits.

- ces importations, en effet, ne se composent plus seulement de "consommations intermédiaires", mais aussi, dans une proportion de plus en plus forte, de produits finis (montres complètes). C'est le signe que le marché suisse s'ouvre aux produits finis étrangers, en particulier aux montres à quartz à affichage digital de bas prix, soit qu'il s'agisse de produits assemblés à l'étran- 
Tableau $n^{\circ} 1$

\section{IMPORTATIONS HORLOGERES SUISSES}

\begin{tabular}{|c|c|c|c|c|}
\hline & 1960 & 1970 & 1980 & 1985 \\
\hline En valeur (millions de francs) & 29.80 & 153.60 & 399.50 & 536.60 \\
\hline En $\%$ des exportations horlogéres & 2.3 & 5.8 & 11.3 & 12.4 \\
\hline
\end{tabular}

Par catégories de produits (en \%)

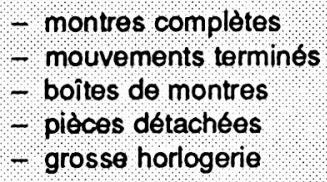

\begin{tabular}{|c|c|c|c|}
\hline 20 & 6.1 & 18.7 & 23, \\
\hline ins. & 0.1 & 51 & 1.5 \\
\hline 27.2 & 26.8 & 36.1 & 32.3 \\
\hline 40.9 & 41.9 & 26.6 & 29.9 \\
\hline 29.9 & 25.1 & 14.4 & 13.1 \\
\hline
\end{tabular}

Principaux pays fournisseurs (en $\%$ )

\begin{tabular}{|c|c|c|c|c|}
\hline - République fédérale d'Allemagne & 51.7 & 42.8 & 23.0 & 19.6 \\
\hline - France & 13.1 & 13,6 & 21.6 & 176 \\
\hline - Italie & 22.1 & 20.6 & 22.5 & 16.4 \\
\hline - Hong Kong & 0.7 & 8.2 & 10.3 & 15.3 \\
\hline - Thailande & ins & 0.5 & 1.5 & 8.0 \\
\hline J Japon & 07 & 3.3 & 6.5 & 6.1 \\
\hline - Malaisie & ins. & ins. & 0.6 & 1.7 \\
\hline - Singapour & ins. & 0.5 & 3.5 & 1.6 \\
\hline - Etats-Unis & 2.3 & 6.4 & 12 & 1.4 \\
\hline - Grande-Bretagne & 17 & 2.5 & 2.2 & 1.2 \\
\hline - Taiwan & ins. & ins. & 1.6 & 0.9 \\
\hline - Philippines & ins. & ins. & 0.3 & 07 \\
\hline - République Popularre de Chino & ins. & ins. & ins & 0.4 \\
\hline - Corée du Sud & ins. & ins. & 0.2 & 0.2 \\
\hline
\end{tabular}

Note. ins. (insignifiant) = inférieur a $0.1 \%$

Source: Statistique annuelle du commerce extérieur de la suisso 
ger pour le compte de fabricants suisses d'horlogerie, soit qu'il s'agisse de produits concurrents importés en Suisse par des maisons de distribution (grandes surfaces, magasins de "discount», etc.)

- dans ce contexte, la part prise par les NPI asiatiques à ces livraisons - ainsi que par d'autres pays de la région qui eux aussi cherchent à développer leurs exportations d'articles manufacturés (la Thaïlande, la Malaisie, les Philippines, la République Populaire de Chine par l'intermédiaire de ses «zones économiques spéciales ....)-devient de plus en plus forte et représente globalement $29 \%$ de la valeur totale des importations horlogères suisses en 1985. On relèvera en particulier la progression de Hong Kong qui fournit à la Suisse, dans le détail, la plus grande partie - les deux tiers, soient près de 4 millions de pièces en 1985 - de ses importations de montres complètes, de celles de mouvements terminés - second fournisseur derrière le Japon -, de celles de pièces détachées second fournisseur après la France - et enfin de celles de boîtes de montres - plus de 3 millions de pièces. Lorsque l'on sait que le prix unitaire moyen des montres importées de Hong Kong est de Frs. 2.30 environ en 1985, cette progression se comprend facilement! A signaler aussi le rôle de plus en plus important joué depuis les années 1980 par la Thaïlande qui fournit à l'industrie horlogère suisse une quantité croissante de pièces détachées et de boîtes de montres. Bref, et même si ces données par définition «anonymes" ne permettent pas de déterminer dans quel cadre se font ces échanges (entre entreprises indépendantes? dans le cadre d'accord de sous-traitance? entre filiales d'un même groupe?), il apparaît déjà que les industriels et distributeurs horlogers suisses ne sont évidemment pas restés insensibles aux avantages que représentait la production horlogère en Asie du Sud-Est.

\section{L'épuisement des technologies traditionnelles}

L'étude de l'évolution des exportations horlogères suisses au cours de cette même période permet de souligner d'autres phénomènes même si, à première vue, ces exportations paraissent rester relativement stables dans leur répartition, que ce soit par catégories de produits ou par pays de destination (voir tableau 2 en page suivante). Ainsi les montres complètes demeurent-elles de 1970 à 1985, et de loin, la première catégorie de produits exportés par la Suisse (plus de $70 \%$ de ses exportations en valeur). De même, et si l'on excepte quelques modifications dues pour une bonne part à l'évolution des cours de change, les dix principaux clients de l'industrie horlogère suisse restent globalement les mêmes au cours de cette période, soit un certain nombre de grands pays industrialisés (Etats-Unis, RFA, Italie, France, Japon, Grande-Bretagne), deux pays à revenus pétroliers (Arabie Saoudite et Emirats arabes unis), ainsi que les deux plaques tournantes commerciales (Hong Kong et Singapour) qui, en Asie du Sud-Est, jouent depuis plusieurs décennies déjà le rôle de marché de redistribution pour les produits horlogers suisses.

En fait, le changement est plus subtil et concerne le contenu et la destination des exportations suisses de pièces détachées horlogères, parmi lesquelles des 
mouvements non assemblés sous la forme d'ébauches ou de chablons.

En 1970, en effet, les livraisons suisses de pièces détachées étaient relativement faibles encore $-6,4 \%$ de la valeur totale des exportations horlogères suisses - et principalement orientées vers d'autres pays horlogers (RFA, France, Japon, Etats-Unis, Grande-Bretagne, Italie) au sein desquels les horlogers suisses ont d'ailleurs implanté, comme nous l'avons noté, des unités de production. Elles étaient donc destinées à alimenter soit les filiales de sociétés suisses, soit des fabriques indépendantes clientes de l'horlogerie suisse. Et seul, parmi les pays en développement, le Mexique importe alors une quantité significative de ces fournitures, phénomène qui s'explique par la présence dans ce pays, dès la fin des années 1960, d'une fabrique d'horlogerie liée à des intérêts suisses.

Puis, jusqu'en 1980, ce mouvement d'exportations de pièces détachées se développe fortement pour représenter $11,9 \%$ de la valeur des exportatios horlogères suisses cette année-là. On constate alors que les exportations d'ébauches et chablons jouent un rôle décisif dans cette progression puisque leur nombre passe de 2 à près de 33 millions de pièces de 1970 à 1980, leur valeur globale de 21 à 189 millions de francs. II s'agit en fait pour plus des $90 \%$ de mouvements de montres non assemblés de technologie mécanique, qui sont destinés en proportion croissante à un certain nombre de pays en développement: Hong Kong qui, à elle seule, en absorbe près de $80 \%$ en nombre de pièces, mais aussi le Brésil, Singapour, la Corée du Sud, le Mexique, le Nigéria et l'Inde, etc.

Ce qui indique que les horlogers suisses, et en tout premier lieu les fabricants de montres de type aroskopf - ou montres mécaniques de conception et de fabrication élémentaires - trouvent dans le développement de l'industrie horlogère de ces pays un certain substitut à la baisse de leurs exportations de produits terminés. Au cours de cette même période - 1970-1980 -, les exportations suisses de montres et mouvements terminés de type roskopf passent en effet de plus de 32 à 15 millions de pièces. Ce sont là les principales «victimes» de la mutation technologiue et de la concurrence représentée par la fabrication de plus en plus massive de montres à quartz à affichage digital.

La croissance de ces livraisons d'ébauches et chablons de type roskopf, qui culminera en 1980, correspond donc au transfert progressif d'une technologie parvenue à «maturitén et pour laquelle la fabrication en pays à bas salaires présente encore un certain avantage. On distinguera toutefois le cas de pays tels que le Brésil, le Nigéria ou le Mexique, dont la production de montres mécaniques est principalement destinée au marché intérieur, de celui de Hong Kong, Singapour, ou de la Corée du Sud, dont la production de montres mécaniques de bas de gamme, à partir de fournitures suisses, sert avant tout à l'exportation.

Ainsi est rendu possible, à Hong Kong en premier lieu, le rapide essor d'une production locale qui, après s'être limitée à la fabrication de pièces de l'habillement dans les années 1960 (boîtes de montres, bracelets, etc.), trouve là l'occasion d'évoluer vers l'assemblage de montres et mouvements proprement dit.

Dès 1980, cependant, les exportations suisses de pièces détachées se réduisent rapidement - de 424 millions de francs en1980 à 267 millions en 1983 -, le nombre d'ébauches et chablons exportés par la Suisse passant en particulier de 
Tableau $n^{\circ} 2$

\section{EXPORTATIONS HORLOGERES SUISSES}

\begin{tabular}{|c|c|c|c|c|}
\hline & 1970 & 1975 & 1980 & 1985 \\
\hline En valeur (millions de francs) & 2629.0 & 3141.3 & 3549.9 & 4311.2 \\
\hline
\end{tabular}

Par catégories de produits (\%)

- montres complètes
- boîtes de montres tres
- piéces détachées
- grosse horlogerie

\begin{tabular}{|c|c|c|c|}
\hline 77.3 & 76.1 & 70.6 & 78.9 \\
\hline 12.5 & 10.5 & 11.6 & 5.4 \\
\hline 1.2 & 2.7 & 3.2 & 2.4 \\
\hline 6.4 & 7.9 & 11.9 & 10.4 \\
\hline 2.6 & 2.8 & 2.7 & 2.9 \\
\hline
\end{tabular}

Principaux pays de destination (\%)

\begin{tabular}{|c|c|c|c|c|}
\hline - Etats-Unis & 19.6 & 13.0 & 12.0 & 18.3 \\
\hline - Hong Kong & 9.4 & 9.1 & 15.9 & 11.6 \\
\hline - République Fédérale d'Allemagne & 7.2 & 8.1 & 8.9 & 8.4 \\
\hline Italie & 6.4 & 6.9 & 8.3 & 7.9 \\
\hline France & 3.2 & 5.1 & 6.6 & 6.5 \\
\hline - Arabie Saoudite & 0.7 & 2.7 & 5.7 & 4.7 \\
\hline Japon & 4.3 & 5.9 & 3.6 & 4.6 \\
\hline Grande-Bretagne & 5.7 & 6.5 & 4.0 & 4.5 \\
\hline Singapour & 1.8 & 19 & 2.5 & 3.2 \\
\hline Emirats arabes unis & 3.5 & 1.9 & 2.5 & 2.2 \\
\hline Thailando & ins. & ins. & 0.1 & 1.4 \\
\hline Brésil & 1.8 & 1.5 & 1.4 & 1.3 \\
\hline - République Populaire de Chine & 0.8 & 0.9 & 1.1 & 1.3 \\
\hline Mexique & 2.0 & 1.2 & 1.1 & 0.5 \\
\hline Taiwan & ins. & 0.3 & 0.8 & 0.5 \\
\hline - Philippines & ins. & 0.1 & 0.2 & 0.4 \\
\hline Indo & 0.1 & 0.1 & 0.3 & 0.4 \\
\hline Malaisie & ins: & 01 & 0.2 & 0.3 \\
\hline - Coróo du Sud & ins. & ins. & ins. & 0.2 \\
\hline
\end{tabular}

Noto. ins . (insignifiant) = inférieur a $0.1 \%$

Source: Statistique annuelle du commerce extérieur de la Suisse 
près de 33 millions de pèces en 1980 à 12,6 millions en1983, avant de connaître depuis cette date une certaine reprise - 18,8 millions de pièces en 1985.

II faut voir dans ce «passage à vide " la conséquence du retard avec lequel l'industrie horlogère suisse dans son ensemble s'est adaptée aux nouvelles technologies: alors que ses exportations de fournitures liées à la fabrication de montres mécaniques chutent, elles ne sont que lentement remplacées par des exportations de fournitures électroniques, d'ébauches et chablons en particulier. Ce n' est en effet que depuis quelques années que l'horlogerie suisse se montre capable de rivaliser, quoique dans une faible mesure seulement, avec les principaux groupes horlogers japonais (Hattori-Seiko, Citizen Watch, Casio...) dans l'approvisionnement des marchés horlogers du Sud-Est asiatique. Un seul coup d'œil porté sur les importations horlogères de Hong Kong pour l'année 1985 montre en effet à quel point les horlogers japonais, qui exportent cette année pour plus de 2,7 milliards de dollars Hong Kong - 346 millions US $\$$ - dans la colonie britannique de fournitures d'horlogerie de petit volume (mouvements de montres assemblés, boîtes, affichages à cristaux liquides, pièces détachées...), ont nettement pris le dessus dans ce domaine sur les horlogers suisses -478 millions de dollars Hong Kong ou 61 millions US\$(4).

Reste le fait que, depuis 1983, les livraisons suisses de pièces détachées horlogères à destination de certains pays du Sud-Est asiatique montrent une nette croissance - dans le cas de la Thaïlande, de la Malaisie et des Philippines -, qu'elles marquent une certaine reprise à destination de Hong Kong - elles s'élèvent à 96 millions de francs en 1985 contre 63 millions en1982 -, alors qu'elles tendent à stagner, voire à baisser, dans le cas d'autres pays - la République Populaire de Chine, Singapour, Taiwan et la Corée du Sud. Ainsi toute une géographie des exportations suisses de fournitures horlogères - nous nous sommes limités ci-dessus aux pièces détachées proprement dites sans y inclure les mouvements de montres terminés et les boîtes de montres - paraît se mettre en place et tend à indiquer que, hormis Hong Kong qui reste en valeur absolue le marché le plus important pour ce type d'exportations, de nouveaux lieux de production - la Thaïlande, la Malaisie, les Philippines, appartenant à ce que certains ont appelé la seconde vague de pays exportateurs de produits manufacturés - commencent à compter.

Certes, comme le montrait le tableau 2, leur importance en tant que marchés dans le cadre des exportations horlogères suisses prises dans leur ensemble peut paraître mineure - 60 millions de francs ou $1,4 \%$ du total dans le cas de la Thaïlande par exemple. Par contre, si l'on considère que ces nouveaux lieux de production ont comme fonction d'effectuer des opérations telles que l'assemblage de composants horlogers et de mouvements, ou encore la fabrication de boîtes pour le compte d'entreprises horlogères suisses, leur rôle est loin d'être négligeable puisqu'il permet de réduire les coûts de production de montres électroniques de bas de gamme.

II n'est pas inutile en effet de rappeler ici que l'industrie horlogère suisse n'a cessé de se concentrer depuis le milieu des années 1970 sur les produits de haut de gamme et de luxe en perdant peu à peu sa vocation de producteur de masse 
dans le bas de gamme horloger. De 1974 à 1982, ses exportations de montres complètes passent en effet de 59 à 18,5 millions de pièces, ses exportations de mouvements terminés de 25,4 à 12,7 millions de pièces. Le fait que cette industrie soit parvenue depuis quelques années à infléchir ce mouvement et à exporter à nouveau en quantités croissantes des mouvements non assemblés, ébauches et chablons ainsi que d'autres fournitures, à destination de pays en voie de développement, n'est donc pas sans importance et ne paraît nullement contradictoire avec la priorité donnée, par la SMH notamment (Société suisse de Microélectronique et d'Horlogerie), première concentration horlogère suisse, à la relance des exportations de produits finis sur le lieu de production suisse, à l'image de la «Swatch».

L'écoulement de fournitures horlogères dans un certain nombre de pays du Tiers monde, qu'ils représentent des marchés intérieurs considérables comme le Brésil, l'Inde et la Chine, ou qu'ils constituent en quelque sorte des bases d'exportations comme Hong Kong ou la Corée du Sud, permet en effet de produire en Suisse des composants en plus grandes séries, et par là de rentabiliser dans des conditions plus profitables les équipements qui servent à leur fabrication. Dans la concurrence qui oppose les principaux groupes horlogers à l'échelle mondiale, d'autres moyens servent également à cet objectif - la diversification de la production en dehors de la seule horlogerie... ou encore la volonté de faire fonctionner ces équipements de manière continue, vingt-quatre heures sur vingt-quatre... (5) -, mais ils ne paraissent pas antagoniques à la poursuite d'une politique soutenue d'approvisionnement des industries horlogères qui se développent dans le Tiers monde.

\section{La présence des horlogers sulsses en Asle du Sud-Est}

\section{Une implantation relativement faible et dispersée}

Ceci dit, l'intérêt représenté par les NPI asiatiques ne s'est évidemment pas limité à cette dimension purement commerciale et la possibilité de recourir à une maind'œuvre jeune, en majorité féminine, bon marché et pouvant être rapidement formée à des tâches relativement peu qualifiées, a très tôt représenté un attrait supplémentaire. Le passage à la fabrication de montres électroniques a eu en effet comme conséquence, dans un premier temps du moins, "d'utiliser des composants dont la production a pu être suffisamment simplifiée pour être menée à bien dans des pays à bas salaires d'Asie du Sud-Est $\nsim(6)$. Dans un premier temps seulement puisque, ensuite, certaines opérations telles que le "bonding" des circuits intégrés - qui consiste à les fixer sur un substrat - ont été automatisées pour les plus grandes séries.

En conséquence, et c'est là qu'il s'agit de distinguer des pays tels que l' Inde du cas particulier des NPI asiatiques, ce n'est plus tellement la pénétration d'un marché plus ou moins fermé aux importations horlogères qui était visée, mais bien l'utilisation d'une force de travail peu rétribuée et souvent fortement «disciplinée». S'ajoutait à cet élément d'autres facteurs tels que, comme dans le cas de Hong 
Kong, l'existence des infrastructures nécessaires à une activité industrielle, la très grande liberté accordée à la circulation des capitaux ou encore l'absence de toute taxe à l'importation. D'où la mise en place par les firmes horlogères d'une stratégie dite de ufragmentation " (des diverses filières de production) et de "délocalisation" (de certaines opérations) en pays à bas salaires, stratégie que les principaux groupes horlogers japonais (ceux que nous avons déjà cités) et nord-américains (Timex, Bulova...) ont été parmi les premiers à appliquer.

Mais qu'en est-il en ce qui concerne l'industrie horlogère suisse?

Dès le milieu des années 1970, une enquête réalisée par l'OFIAMT (Office fédéral de l'Industrie, des Arts et Métiers et du Travail) auprès des milieux patronaux horlogers révélait une très forte sensibilité à la perspective de transferts de production à l'étranger: plus de la moitié des entreprises et experts interrogés déclaraient que de tels transferts étaient « relativement probables» ou "probables" pour l'assemblage des montres mécaniques ainsi que pratiquement pour l'ensemble de la productiondes montres à quartz à affichage digital, de l'assemblage des pièces détachées à celui du produit fini (7).

Prévisions qui semblaient se confirmer puisqu'en automne 1978, les deux principales concentrations horlogères suisses - l'ASUAGet la SSIH (Société Suisse pour l'Industrie Horlogère) - annonçaient coup sur coup leur décision de déplacer une parte de leur production - l'assemblage de mouvements de montres à affichage digital et la fabrication de boîtes de montres respectivement - en Asie du Sud Est.

N'allait-on donc pas vers un mouvement massif de transfert de la production horlogère suisse à l'étranger?

Un bilan de ce mouvement, quoique limité ici au seul cas de Hong Kong, invite pourtant à une certaine réserve. Certes, il peut être établi que pratiquement toutes les principales firmes et concentrations horlogères suisses ont cherché à un moment ou à un autre à s'implanter sous une forme industrielle dans la colonie britannique: c'est le cas en particulier du groupe ASUAG (qui crée en 1978, nous l' avons dit, une entreprise conjointe avec l'un des principaux groupes industriels locaux), ou de la SSIH par le biais de sa division de produits économiques - Economic Swiss Time-, de la SGT (Société des Garde-Temps) aujourd'hui disparue, de Mondaine Watch, de Baumgartner Frères SA, de Ronde SA, etc. (8).

Toutes ces initiatives montrent donc qu'il a bel est bien existé au sein de l'industrie horlogère suisse, si l'on excepte les fabricants de produits de très haut de gamme pour qui le problème ne se posait pas, une réelle volonté de «multinationalisation \# de leurs activités jusque-là localisées en pays industrialisés.

Cependant, comme le montre le tableau en page suivante, la somme des investissements directs réalisés par des firmes suisses dans I 'industrie horlogère de Hong Kong restait à fin 1983 d'un montant relativement faible-27,7 millions HK\$ ou environ 9 millions de francs suisses - et surtout nettement inférieur - près de neuf fois - au montant des investissements directs réalisés par les groupes horlogers japonais.

II convient cependant de noter au passage que l'ensemble de ces investissements - 389,7 mios HK\$ à fin 1983 - ne correspondent alors, si l'on peut dire, qu'à 
47 établissements industriels occupant environ 8.800 personnes, ce qui ne représente qu'une faible partie du nombre des établissements horlogers recensés à Hong Kong - plus de 1.500 - ainsi qu'à moins d'un quart des effectifs globaux de la branche-38.000 personnes environ - (9). Quelques années plus tôt, pourtant, cette présence étrangère était proportionnellement beaucoup plus forte puisqu'elle correspondait en 1974 à plus de $70 \%$ des effectifs employés dans l'industrie horlogère locale. Ce qui montre, en d'autres termes, que la croissance très rapide connue par la production locale dès le milieu des années 1970, production qui se concentre alors sur la fabrication massive de montres digitales économiques, est due avant tout à $l$ 'initiative des entrepreneurs locaux, chinois dans leur immense majorité, qui se montrent très prompts à s'adapter à ce type de production, même s'ils restent largement dépendants à l'égard de fournisseurs étrangers en ce qui concerne leur approvisionnement en composants et autres produits semi-finis.

Tableau $n^{\circ} 3$

\section{INVESTISSEMENTS ETRANGERS REALISES DANS LINDUSTRIE HORLOGERE A HONG KONG A FIN 1983 (mlos HK\$)}

Pays dorigine

Montant

En \% du total

Japon

Royaume Uni

SUISSE

Bermudes

Etats-Unis

Autres
240.0

72.9

27.7

26.9

4.3

5.4
61.6

18.7

71

6.9

0.5

1.4

Total

389.7

100

Source. Hong Kong Government Industry Department

Ceci précisé, il reste que jusqu'aux premières années 1980, la présence des horlogers suisses à Hong Kong sous la forme d'unités de production apparaît, au vu du rôle commercial très important joué par l'horlogerie suisse dans le développement de l'industrie locale, comme relativement faible par rapport à celle de leurs concurrents japonais. Quant à la présence de ces mêmes horlogers dans les autres 
NPI asiatiques, elle apparaît elle-aussi, quand bien même nous n'en avons pas fait l'étude systématique, nettement en deçà de ce que l'on pouvait «attendre» compte tenu de la position de l' industrie horlogère suisse au début des années 1970.

\section{La persistance de structures dépassées}

A ce paradoxe apparent il existe bien une explication. Après avoir rappelé que dès la fin des années 1960 la Fédération Horlogère Suisse avait, par le biais d'un rapport d'experts, esquissé une stratégie non plus uniquement commerciale mais aussi industrielle, M. Gérard Bauer, ancien président de cette association professionnelle, déclarait: «Elle (cette stratégie) ne fut pas suivie par une mise enceuvre, dans les faits et en temps utile, d'une stratégie industrielle transnationale appliquée, en particulier, aux pays nouvellement industrialisés, analogue à celle réalisée par nombre d'autres secteurs industriels suisses. Cette constatation apparaît d'autant plus surprenante et paradoxale si l'on se souvient que l'horlogerie suisse a été longtemps la seule industrie helvétique à avoir détenu une part aussi importante des marchés mondiaux. II faut rechercher les raisons de ce divorce entre les réflexions, le diagnostic précité et la thérapeutique négligée dans les faits, dans la confiance surannée en un monopole industriel au XXe siècle, l'esprit qui avait inspiré les anciennes structures cartellaires horlogères et qui survivait encore après leur trop lente disparition, l'absence de vue d'ensemble persistante guidant la conception et la conduite des affaires horlogères, chacun des secteurs de cette industrie ne prenant que trop souvent en considération que ses seuls intérêts, et à court terme. (10)

Tout au long des années 1970, en effet, l'industrie horlogère suisse conserve, malgré un certain nombre de concentrations et la disparition de plusieurs centaines d'entreprises, les structures qui étaient les siennes au cours des décennies précédentes. Caractérisées par une nette coupure entre le secteur des fabricants de pièces constitutives et celui des simples établisseurs ou fabricants de produits finis, ces structures font que la production horlogère suisse demeure dispersée en un grand nombre de fabriques et partagée en de nombreux centres d'intérêts (les fabricants de pièces détachées, les fabricants de pièces de l'habillement, les fabricants de montres roskopf, etc.) sans qu'une politique commune ne puisse réellement être mise en œuvre. A cela s'ajoute le fait que les principales concentrations horlogères du moment (ASUAG, SSIH) restent de nature essentiellement financière alors que se maintenaient, en leur sein, ce que le Professeur Gœtschin appelait des «baronnies»: «En fait, comme à la SSIH ou à l'ASUAG, on a créé des fédérations d'entreprises familiales, avec leurs baronnies. Chacun conserve ses prérogatives et gère ses affaires comme autrefois, lorsqu'elles étaient indépendantes. (11).

Le maintien de telles structures, avec tout ce que cela implique, est alors lourd de conséquences puisque, au fait que nous avons déjà évoqué - l'absence d'une politique concertée en matière d'implantation industrielle à l'étranger -, s'ajoute le retard pris par l'horlogerie suisse dans son ensemble en matière d'acquisition et d'application des nouvelles technologies sous la forme de grandes séries. «Une 
entreprise de deux cents personnes, avec 30 ou 40 millions de chiffre d'affaires, n'avait pas la masse critique pour attaquer les marchés, pour maîtriser l'évolution de la technologie et pour s'occuper de la formation des cadres... , ajoutait M. Gœtschin.

Pour notre part, et ceci nous paraît beaucoup plus important, on ajoutera que les conséquences les plus lourdes sont sans doute à chercher dans la disparition de plus de 60.000 emplois de 1970 à aujourd'hui dans des régions dont la production horlogère était pratiquement la seule activité industrielle, sans que l'on ait pris garde à l'époque à diversifier leurs activités et préparer le passage aux nouvelles technologies. Si certains de ces emplois disparaissent en raison des mesures de rationalisation prises au cours de cette période, il n'en reste pas moins que, depuis le milieu des années 1970, c'est bel et bien la perte de compétitivité de la production horlogère située en Suisse et la lenteur avec laquelle se diffusent les nouvelles technologies de production qui se trouvent à l'origine de la majorité de ces pertes d'emplois. Quant à la «concurrence du Sud-Est asiatique», souvent présentée comme ayant été la cause première du déclin de l'industrie horlogère suisse, elle n'a pu réellement se développer, comme nous l'avons montré, que grâce aux fournitures horlogères que la Suisse, en particulier, lui a assurées dans un premier temps... puis grâce au retard accumulé par ces mêmes horlogers suisses en matière de fabrication de produits électroniques.

En attendant, la situation des premières années 1970 se prolonge jusqu'au tournant des années 1980, période où les groupes ASUAG et SSIH enregistrent des résultats de plus en plus déficitaires. Suivent alors, à l'initiative des grandes banques helvétiques, plusieurs mesures d'assainissement avant que les deux groupes ne fusionnent et ne soient repris depuis 1985 par des investisseurs privés.

Globalement, la restructuration de l'industrie horlogère suisse n'est donc intervenue que de manière tardive et c'est ce qui explique, sans naturellement les ajustifier» pour autant, les décisions prises au cours des années 1982-86: «dégraissage» des activités les moins rentables; fermetures d'entreprises; placement de manufactures horlogères jusque-là quasi indépendantes (Omega, Longines) sous la tutelle du groupe ETA, fabricant de mouvements et composants horlogers; forte réduction du nombre des «calibres» - ou types de mouvements -; réduction du nombre de marques; etc.

La priorité tout au long de cette phase décisive de la restructuration de l'industrie horlogère suisse est alors manifestement mise sur la rentabilisation, par le développement entre autres de l'automation, de l'appareil de production situé en Suisse, afin d'en faire un instrument de areconquête» des marchés horlogers, y compris du marché de la montre économique (la «Swatch $*$ ), tout en cherchant à développer les activités extra-horlogères en prenant pied dans d'autres secteurs de l'électronique (les télécommunications, les appareils de mesure ou la robotique). L'accord rendu public en juin dernier entre le groupe SMH et le groupe Philips, premier producteur européen de circuits intégrés, s'inscrit d'ailleurs parfaitement dans le cadre de cette stratégie... tout comme l'insistance avec laquelle les nouveaux patrons du premier groupe horloger suisse cherchent à introduire le travail en continu dans certains de leurs ateliers. 
A noter également au cours de cette période la décision par les groupes ASUAG et $S S I H$, prise alors dans la tourmente des mesures d'assainissement, d'abandonner les activités qu'ils avaient transférées sous une forme directe en Asie du Sud-Est à la fin des années 1970: la Modutek Ltd., entreprise conjointe établie entre Ebauches Electroniques SA (du groupe ASUAG) et le groupe industriel de Hong Kong Stelux Manufacturing Co., cesse ses activités d'assemblage de mouvements de montres digitales en octobre 1982; Precision Watchcase Ltd., fabrique de boîtes de montres créée à Singapour par la $S S I H$ et un groupe industriel japonais, fait de même quelques mois plus tard; la société Economic Swiss Time Hong Kong, filiale du groupe SSIH chargée dès 1978 d'effectuer toutes les activités de production de montres économiques, est vendue en 1983 à son ancien directeur.

Est-ce à dire, pour autant, que la politique d'implantation industrielle à l'étranger, et en particulier dans les pays en développement, soit abandonnée? Certainement pas. Tout d'abord parce que l'industrie horlogère suisse ne se limite évidemment pas au seul groupe SMH et que d'autres entreprises ont cherché depuis longtemps à axer leur développement sur ces pays. D'autre part, et ceci doit aussi être valable pour le groupe $S M H$, parce que la possibilité d'écouler des composants horlogers auprès des principaux marchés de transformation du SudEst asiatique (Hong Kong bien sûr, mais aussi la Corée du Sud et Taïwan), de réaliser des économies en coûts de production dans des pays où les salaires sont encore plus bas en y faisant notamment appel à des entreprises de sous-traitance (en Thaillande, en Malaisie ou aux Philippines, pays dont nous avons vu que l'importance en tant que débouchés et fournisseurs de la Suisse n'avait cessé de croître depuis quelques années), ou encore de parvenir à pénétrer d'importants marchés intérieurs (on pense ici à la Chine ou à l'Inde), ne paraît nullement incompatible, comme nous l'avons déjà mentionné, avec l'application d'une stratégie de rationalisation de l'appareil de production situé en Suisse.

\section{Pour conclure}

Il y a quelques années encore le mouvement de «délocalisation* de la production horlogère en direction de pays à bas salaires, en Asie du Sud-Est en particulier, paraissait, comme nous le notions en introduction, se poursuivre de manière quasi irréversible. N'était-ce pas la la conséquence logique de ce qu'un nombre croissant d'opérations productives avaient été considérablement simplifiées avec le passage à la micro-électronique et pouvaient dorénavant être effectuées par une maindoeuvre peu qualifiée? En conséquence n'était-il pas dans l' intérêt des firmes horlogères originaires des pays industrialisés, de Suisse en particulier, de transférer massivement leurs activités en Asie du Sud-Est et de profiter ainsi d'une main-d'œuvre à bon marché?

Aujourd'hui, et quand bien même la perspective de nouvelles délocalisations n'est pas à écarter, plusieurs éléments, que nous n'avons fait qu'effleurer dans le cadre de cet article, obligent à se montrer plus prudent à ce sujet; en voici pour conclure une brève présentation: 
1) C'est premièrement, d'un point de vue général, l'évolution des techniques de production horlogère, permettant aujourd'hui, grâce à la robotique et la mise en place d'ateliers de fabrication intégrés et automatisés, de maintenir ou de rapatrier un certain nombre d'opérations en pays industrialisés, alors que celles-ci avaient fait l'objet d'un transfert de production en pays à bas salaires au cours des années 1970. D'autre part, le fait que la plus grande part de la consommation horlogère mondiale reste concentrée dans les pays industrialisés implique également la nécessité d'être présent dans ces marchés.

2) Deuxièmement, le mouvement de redistribution géographique des activités horlogères n'a pas seulement obéi à l'intérêt de firmes transnationales - ou en voie de l'être - mais aussi à celui d'un capital industriel en voie de formation dans les NPI asiatiques. A l'exception de Singapour où la présence des investissements directs étrangers est largement prédominante mais où, et ceci explique cela, la production horlogère ne s'est que peu développée, la croissance de l'industrie horlogère dans les NPI asiatiques a avant tout reposé dès le milieu des années 1970 sur l'initiative locale.

Le cas est patent à Hong Kong où, commenous l'avons brièvement signalé, la croissance très rapide de la production de montres électroniques à affichage digital est manifestement due, dès 1975, au dynamisme des entreprises locales, dont le nombre passe en quelques années seulement de 300 à plus de 1.500. Que la plupart de ces entreprises se soient limitées à des opérations d'assemblage et qu'elles paraissent travailler le plus souvent en tant que soustraitants pour le compte de firmes horlogères étrangères ou pour celui de grandes sociétés de distribution, ceci n'enlève rien au fait que cette industrie ait acquis et renforcé une certaine autonomie à l'égard des firmes transnationales de la branche.

Très vite d'ailleurs, c'est-à-dire dès les années 1981-82, la production locale de montres économiques et de technologie élémentaire atteint des quantités considérables et contribue fortement à la situation de surproduction qui caractérise alors les marchés horlogers. Or les conséquences d'une telle situation - l'accumulation des stocks à tous les niveaux de la distribution horlogère, l'effondrement rapide des prix des produits horlogers de bas de gamme et la réduction des marges de profits - sont alors loin de ne frapper que les seuls fabricants locaux, comme l'indiquent les difficultés rencontrées à cette époque par les groupes horlogers japonais, eux aussi lancés dans une production de plus en plus pléthorique. Si l'émergence des NPI dans le SudEst et l'Est asiatique a donc pu constituer une opportunité, dans un premier temps, pour les firmes originaires des pays industrialisés en termes de localisations industrielles, elle a aussi conduit, de par sa propre dynamique, à une situation contraire aux intérêts de ces mêmes firmes.

3) S'ajoute à ce qui précède le fait que le développement de l'industrie horlogère parmi d'autres activités manufacturières a conduit dans les NPI asiatiques à une situation proche du plein emploi et, en conséquence, à une pression bien 
légitime à la hausse des salaires locaux et à une amélioration des conditions de travail, même si les salaires pratiqués dans ces pays restent en valeur absolue nettement inférieurs à ceux qui se pratiquent en pays industrialisés et si les conditions de travail y demeurent le plus souvent précaires.

4) Concurrence de plus en plus "sauvage" de la part des industriels locaux et hausse relative des salaires ont donc conduit à une certaine détérioration des "avantages comparatifs" détenus par les NPI asiatiques. Les firmes horlogères transnationales, ou de moins les plus importantes d'entre elles, ont alors tout avantage, en situation de très vive concurrence et de désorganisation des marchés, à investir dans la rationalisation de leur production, la fabrication de nouveaux composants plus performants et la sortie de nouveaux produits qui puissent se différencier par leur qualité de l'abondance des produits horlogers économiques en provenance du Sud-Est asiatique. Politique qui favorise à nouveau la localisation de certaines activités en pays industrialisés.

5) Une autre solution consiste aussi pour elles à « relocaliser " certaines de leurs activités dans d'autres pays asiatiques qui se montrent moins «avancés» dans leur développement tout en offrant des conditions d'investissement ou de recours à la sous-traitance plus avantageuses. Mais ceci ne concerne que certaines opérations seulement dans la mesure où le contrôle des activités les plus «stratégiques» (les activités de recherche et de développement technologiques, la fabrication des composants à haute performance) nécessite le plus souvent leur localisation en pays industrialisés. L'accord conclu entre le groupe SMH et Philips visant au développement en Suisse d'une unité commune de production de circuits intégrés paraît s'inscrire, une nouvelle fois, dans cette logique.

6) Ceci ne signifie évidemment pas que l'industrie horlogère au sein des NPI asiatiques soit condamnée à disparaître. Confrontés aux difficultés que nous avons citées précédemment, les industriels horlogers de Hong Kong, à titre d'exemple, cherchent depuis quelques années déjà à y faire face: soit en recourant à de nouveaux lieux de production plus avantageux qui leur permettent de prolonger leur dynamique antérieure - la forte croissance des échanges horlogers entre la colonie britannique et la République Populaire de Chine depuis 1983 tendrait à montrer que les «zones économiques spéciales» chinoises, dont la zone voisine de Shenzhen, jouent dans ce sens un rôle de plus en plus important - soit, pour les entreprises qui en ont les moyens, en intensifiant leur production en capital et en technologie et en la diversifiant vers des produits plus rentables, les montres à quartz à affichage analogique en particulier.

7) Toujours dans le cas particulier de Hong Kong, ceci s'est traduit au cours de ces dernières années par un appel lancé par les fabricants locaux à de nouveaux investissements étrangers: appel qui semble avoir été entendu puisque, 
de 389,7 millions de dollars Hong Kong à fin 1983 , le montant total des investissements directs étrangers dans l' industrie horlogère locale est passé à 603,9 millions à fin 1985 (12). Si cette tendance se confirme, comme semble l'indiquer la diminution, depuis 1981 déjà, du nombre de personnes employées par cette industrie - de plus de 40.000 à 33.000 à fin 1985 -, cela montrerait que l'horlogerie de Hong Kong évolue, en quelque sorte, vers une position intermédiaire entre les lieux où se concentrent aujourd'hui la recherche et le développement des nouveaux produits horlogers - le Japon, la Suisse... - et ceux - les «zones économiques spéciales» en Chine Populaire, la Thaïlande, la Malaisie, les Philippines, et d'autres encore - où s'effectuent les opérations pour lesquelles l'utilisation d'une main-d'œuvre abondante, peu qualifiée et bon marché, reste rentable.

8) Dès lors, et sur la base de ces quelques éléments, il semble bien que le mouvement de «retour» de la production horlogère en pays industrialisés, mouvement que certains ont cru pouvoir observer dans d'autres branches manufacturières (13), n'est pas plus évident que ne le semblait le mouvement inverse de $\propto$ délocalisation $\cdots$ de la production en direction de pays à bas salaires dans les années 1970, et dépend de multiples facteurs. Dans le cas des groupes horlogers japonais, la hausse du yen et l'importance de leur présence industrielle dans les NPI du Sud-Est et de l'Est asiatique a certainement amplifié, au cours de ces dernières années, la tendance au transfert d'opérations de production, de plus en plus intensives en capital et en technologie, en direction de ces pays, mais pour combien de temps? Quant aux industriels horlogers suisses ils continuent à favoriser la rationalisation et le développement de leur appareil de production en Suisse, ce qui ne les empêche pas de recourir de manière croissante à la sous-traitance dans des pays comme la Thaïlande, la Malaisie et les Philippines.

9) Par conséquent, plutôt que d'appréhender la division internationale du travail dans l'horlogerie sous la forme d'une opposition entre "vieux» pays industrialisés, «Nouveaux Pays Industriels» asiatiques et pays qui aspirent à un rôle accru dans la distribution des activités manufacturières mondiales, il nous paraît plus important d'analyser la hiérarchisation qui tend à se renforcer entre les différents stades - et donc les différents lieux - de la production horlogère à l'échelle internationale.

Notes

1. Selon l'appelation utilisée dès 1979 par l'OCDE qui retenait un échantillon de dix pays: Hong Kong, Singapour, Corée du Sud et Taiwan (les quatre NPI asiatiques) ainsi que le Brésil et le Mexique en Amérique latine, la Grèce, le Portugal, l'Espagne et la Yougoslavie en Europe. Cf. OCDE, L'incidence des Nouveaux Pays Industriels sur la production et les échanges des produits manufacturés, Paris, 1979. 
2. D'après Nations Unies, Annuaire statistique du commerce international, groupe 885 de la Classification Type du Commerce International. Révision 2.

3. Selon les estimations établies par la Fédération de l'industrie horlogère suisse (FH).

4. Hong Kong Trade Statistics, année 1985.

5. Cf. à sujet l'article de François Milliet, ancien président du groupe SMH, La nouvelle horlogerie, paru dans la Revue Economique et Sociale, $n^{\circ} 2$, juin 1985, Lausanne.

6. Philippe Aydalot, Dynamique spatiale et développement inégal, Economica (seconde édition), Paris, 1980, p. 324.

7. CF. OFIAMT, groupe de travail «Problèmes des régions horlogères", Enquête dans l'industrie horlogère sur les prévisions relatives aux places de travail, Berne, juin 1976. tableau 3, p. 15.

8. Pour plus de détails, cf. Blanc Jean-François, op. cit, chapitre 5.

9. Hong Kong Industry Department, Statistical Tables, 1984.

10. Gérard Bauer, Point de vue, in: Service d'information Tiers monde, Horlogerie suisse et Tiers monde, Lausanne, mai 1984, pp. 63-64.

11. «24 heures", 17.9.1982.

12. Hong Kong Industry Department, Statistical Tables, 1986.

13. Cf. à ce propos l'étude de J. Rada, La micro-électronique et son impact socio-économique, Bureau International du Travail, Genève, 1982, et l'analyse critique qu'en fait Dieter Ernst dans The Global Race in Microelectronics - Innovation and Corporate Strategies in a Period of Crisis, Campus Verlag, Francfort, 1983, argumentation reprise dans Progrès récents de l'industrie des semi-conducteurs: leurs incidences sur la restructuration technologique et sur les schémas de domination-dépendance dans l'industrie, paru dans: Industrie et Développement, numéro 11, ONUDI, New York, 1985. 